\title{
GLUT4 glucose transporter deficiency increases hepatic lipid production and peripheral lipid utilization
}

\author{
Ko Kotani, ${ }^{1}$ Odile D. Peroni, ${ }^{1}$ Yasuhiko Minokoshi, ${ }^{1,2}$ Olivier Boss, ${ }^{1}$ and Barbara B. Kahn' \\ 1Division of Endocrinology, Diabetes and Metabolism, Department of Medicine, Beth Israel Deaconess Medical Center and Harvard Medical School, \\ Boston, Massachusetts, USA. ${ }^{2}$ Division of Endocrinology and Metabolism, Department of Developmental Physiology, \\ National Institute for Physiological Sciences, Okazaki, Japan.
}

\begin{abstract}
A critical defect in type 2 diabetes is impaired insulin-stimulated glucose transport and metabolism in muscle and adipocytes. To understand the metabolic adaptations this elicits, we generated mice with targeted disruption of the GLUT4 glucose transporter in both adipocytes and muscle (AMG4KO). In contrast to total body GLUT4-null mice, AMG4KO mice exhibit normal growth, development, adipose mass, and longevity. They develop fasting hyperglycemia and glucose intolerance and are at risk for greater insulin resistance than mice lacking GLUT4 in only one tissue. Hyperinsulinemic-euglycemic clamp studies showed a $75 \%$ decrease in glucose infusion rate and markedly reduced 2-deoxyglucose uptake into skeletal muscle (85-90\%) and white adipose tissue (65\%). However, $A M G 4 K O$ mice adapt by preferentially utilizing lipid fuels, as evidenced by a lower respiratory quotient and increased clearance of lipids from serum after oral lipid gavage. While insulin action on hepatic glucose production and gluconeogenic enzymes is impaired, hepatic glucokinase expression, incorporation of ${ }^{14} \mathrm{C}$-glucose into lipids, and hepatic VLDL-triglyceride release are increased. The lipogenic activity may be mediated by increased hepatic expression of SREBP-1c and acetyl-CoA carboxylase. Thus, inter-tissue communication results in adaptations to impaired glucose transport in muscle and adipocytes that involve increased hepatic glucose uptake and lipid synthesis, while muscle adapts by preferentially utilizing lipid fuels. Genetic determinants limiting this "metabolic flexibility" may contribute to insulin resistance and type 2 diabetes in humans.
\end{abstract}

\section{Introduction}

Type 2 diabetes is characterized by insulin resistance in muscle, liver, and adipocytes and impaired pancreatic $\beta$ cell function (1). Resistance to the stimulatory effect of insulin on glucose transport and metabolism in muscle and adipocytes is a defect that occurs early in the development of type 2 diabetes $(2,3)$. A key question for understanding the pathogenesis of type 2 diabetes is whether the insulin resistance in different insulin target tissues results from the same genetic defect that directly interferes with insulin action in each of these tissues or whether there is coordinate regulation by which insulin resistance in one of these target tissues causes metabolic or hormonal changes that secondarily alter insulin action in other tissues.

Recent data establish a theme of crosstalk among insulin target tissues $(4,5)$, but little is known about the importance of metabolic adaptations of tissues to the development of insulin resistance. The liver may be a pivotal site for such adaptations, since it can store (via glycogen synthesis and lipogenesis) or release glucose (via glycogenolysis and gluconeogenesis) and release lipid fuels (as lipo-

Nonstandard abbreviations used: ACC, acetyl-CoA carboxylase; $A G 4 K O$, adipose-specific GLUT4-KO; $A M G 4 K O$, adipose- and muscle-specific GLUT4double KO; BAT, brown adipose tissue; 2-DG, 2-deoxyglucose; G6Pase, glucose-6phosphatase; GINF, glucose infusion rate; Het $K O$, adipose- and muscle-specific GLUT4-double heterozygous KO; HGP, hepatic glucose production; MCP-1, monocyte chemoattractant protein-1; $M G 4 K O$, muscle-specific GLUT4-KO; PAI-1, plasminogen activator inhibitor-1; PEPCK, phosphoenolpyruvate carboxykinase; RQ, respiratory quotient; WAT, white adipose tissue. Conflict of interest: The authors have declared that no conflict of interest exists. proteins). Skeletal muscle, on the other hand, has a great capacity to transition between oxidation of carbohydrate and lipid fuels in response to hormonal, mainly insulin and possibly leptin (6), and substrate signals (7). In the setting of diminished insulin effectiveness on glucose metabolism, the degree of flexibility of tissues in using other fuel substrates as well as the capability of the liver to produce other fuels (e.g., VLDL-triglycerides) might affect the evolution of insulin resistance and the development of $\beta$ cell failure and diabetes. In fact, this "metabolic flexibility" seems key for wholebody energy balance and glucose homeostasis, and it is impaired in insulin-resistant, obese, and type 2 diabetic humans (7).

Recently we generated mice with a defined genetic defect in 1 insulin target tissue to determine the effect on whole-body glucose homeostasis and on insulin action in other insulin target tissues $(8,9)$. We targeted glucose transport because it is the rate-limiting step for glucose metabolism in muscle and adipocytes. GLUT4, the major insulin-regulated glucose transporter, is expressed primarily in skeletal and cardiac muscle and adipocytes, with low-level expression in a few other tissues $(10,11)$. GLUT4 expression is downregulated in adipocytes, while GLUT4 function is impaired in skeletal muscle in most type 2 diabetic patients (2). Adipose tissue is an important endocrine organ, but it utilizes relatively little glucose compared with muscle.

Thus, to understand whether the downregulation of GLUT4 expression selectively in adipocytes in obesity or type 2 diabetes could contribute to the pathogenesis of insulin resistance and also to determine the importance of impaired glucose transport in muscle, we previously used Cre-LoxP gene targeting to knock out GLUT4 selectively from adipose tissue or muscle $(8,9)$. Mice 


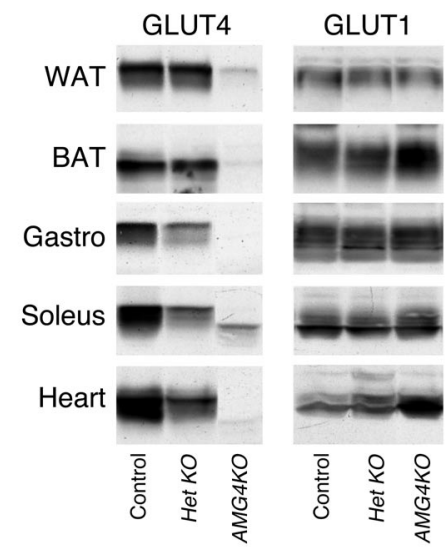

Figure 1

Immunoblot analysis of GLUT4 and GLUT1 from control, Het KO, and $A M G 4 K O$ mice. Tissue extracts from WAT, BAT, gastrocnemius (Gastro), soleus, and heart were prepared and immunoblotted with antibodies for GLUT4 or GLUT1. Shown are representative of 3 different immunoblots performed on 3 separate groups of control, Het $K O$, and AMG4KO mice. $n=17-22$ for control and AMG4KO mice, $n=6$ for Het $K O$ mice.

in which GLUT4 has been reduced in either tissue have glucose intolerance and insulin resistance. Nearly all muscle-specific GLUT4-KO (MG4KO) mice and some adipose-specific GLUT4-KO $(A G 4 K O)$ mice have fasting hyperglycemia, and a subset of $A G 4 K O$ mice develops more severe diabetes. This contrasts with GLUT4null mice, lacking GLUT4 in all tissues, which are growth retarded with cardiomegaly, markedly reduced adipose tissue mass, and shortened lifespan but not frank diabetes (12). However, at least half of the mice that were heterozygous for GLUT4 deletion from all tissues developed overt diabetes by 6 months of age (13).

It was initially surprising to find in $M G 4 K O$ and $A G 4 K O$ mice that, in addition to the tissue that was genetically targeted, the other insulin target tissues also become insulin resistant secondarily (5). In $A G 4 K O$ mice, insulin resistance in the non-genetically targeted tissues appears to be due to a circulating factor, since muscle from these mice responds normally to insulin ex vivo (8).

The current study was designed with 2 major aims. First, to better understand the biological functions of GLUT4, we wanted to determine whether mice lacking GLUT4 in both muscle and adipose tissue would have features of GLUT4-null mice. Second, we sought to determine whether absence of GLUT4 from both adipose tissue and muscle has additive or synergistic effects on glucose homeostasis. Since insulin-stimulated glucose transport is impaired in both tissues in humans with obesity and type 2 diabetes, this would provide important information about the pathogenesis of insulin resistance in these states. Surprisingly, we found in our mice no further impairment in glucose tolerance when GLUT4 was absent from both tissues compared with absence from only one tissue, although the risk for severe insulin resistance was increased. Importantly, the liver appears to partially compensate for the block in glucose transport in muscle and adipose tissue by altering hepatic fuel metabolism, and peripheral tissues adapt by increasing utilization of fatty acids. This model may be informative about the mechanisms underlying type 2 diabetes in humans, since data suggest that lack of this "metabolic flexibility" in obese and type 2 diabetic humans (7) may contribute to the development and progression of insulin resistance.

\section{Results}

Creation and molecular characterization of adipose- and muscle-specific GLUT4-donble KO mice. Mice with KO of GLUT4 simultaneously from both adipose tissue and muscle (aP2-Cre ${ }^{+/-}-\mathrm{MCK}-\mathrm{Cre}^{+/-}$ GLUT4 lox ${ }^{+/+}$) were obtained by breeding aP2-Cre ${ }^{+/+}-\mathrm{GLUT} 4 \mathrm{lox} \mathrm{x}^{+/-}$ mice with $\mathrm{MCK}-\mathrm{Cr}^{+/+}-\mathrm{GLUT} 4$ lox ${ }^{+/-}$mice and identified by PCR using genomic DNA. Homozygous adipose- and muscle -specific GLUT4-double KO (AMG4KO) mice were born with the expected mendelian frequency of $25 \%$ and were indistinguishable from control littermates (carrying aP2-Cre ${ }^{+/-}$and $\mathrm{MCK}-\mathrm{Cr} \mathrm{C}^{+/-}$but not GLUT4 $l o x)$ or adipose- and muscle-specific GLUT4-double heterozygous $\mathrm{KO}($ Het $K O)$ mice at birth.

To confirm the effect of the GLUT4 gene targeting on GLUT4 protein expression, we subjected protein extracts from white adipose tissue (WAT), brown adipose tissue (BAT), skeletal muscle, and heart to Western blot analysis. In Het KO mice, GLUT4 protein levels were slightly reduced in WAT and BAT and reduced by $40-50 \%$ in skeletal muscle and heart (Figure 1, left panels). In AMG4KO mice, GLUT4 protein levels in WAT and BAT were reduced by $70-99 \%$ and those in skeletal muscle and heart were reduced by $98-100 \%$. In Het $K O$ and $A M G 4 K O$ mice, GLUT1 protein levels were normal in WAT and skeletal muscle (Figure 1, right panels). GLUT1 protein levels were increased in BAT and heart of AMG4KO mice.

Impact of absence of GLUT4 in adipose tissue and muscle on growth and body composition. AMG4KO mice developed normally from 3 to 25 weeks of age (Figure 2A). Body weight and body fat content determined by dual-energy $\mathrm{x}$-ray absorptiometry (Figure $2 \mathrm{~B}$ ) were also normal in $A M G 4 K O$ and Het $K O$ mice compared with control littermates at 25 weeks. Body fat assessed as percent of total body weight was normal in $A M G 4 K O$ mice (data not shown).

Impact of absence of GLUT4 in adipose tissue and muscle on glucose tolerance, insulin tolerance, and plasma insulin levels. To determine the physiological consequence of the absence of GLUT4 in AMG4KO mice, we performed glucose tolerance and insulin tolerance tests. Fasting blood glucose was elevated and glucose tolerance was impaired in both female (Figure $3 \mathrm{~A}$ ) and male (not shown) $A M G 4 K O$ mice as early as 2 months of age. In Het $K O$ mice at this age, fasting glucose and glucose tolerance were normal. AMG4KO mice at 2 months of age were very insulin resistant as evidenced
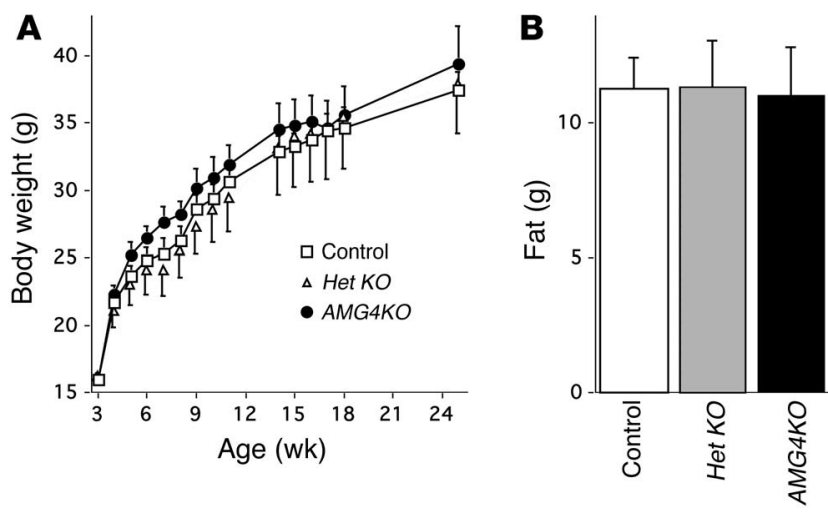

Figure 2

Body weight and fat content of $A M G 4 K O$ mice. (A) Growth curves of female control (open squares), Het $K O$ (open triangles), and $A M G 4 K O$ (filled circles) littermates. (B) Dual-energy $\mathrm{x}$-ray absorptiometry analysis of body fat content in 5- to 6-month-old female control, Het KO, and AMG4KO mice. $n=5-6$ per group. 


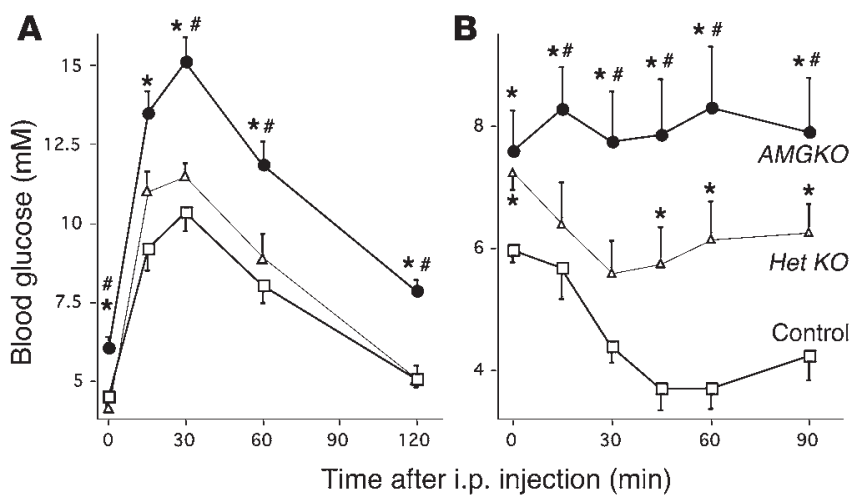

Figure 3

AMG4KO mice have glucose intolerance and insulin resistance. (A) Glucose tolerance tests were performed after 16-hours fasting on 2-month-old female control (open squares), Het $K O$ (open triangles), and $A M G 4 K O$ (filled circles) mice. Animals were injected i.p. with $1 \mathrm{~g} \mathrm{D}$-glucose/kg body weight. Blood glucose was measured immediately before injection and $15,30,60$, and 120 minutes after injection. $n=9-17$ per group. (B) Random-fed, 2-month-old mice were injected intraperitoneally with $0.75 \mathrm{U} / \mathrm{kg}$ body weight of human regular insulin. Blood glucose was determined in the tail vein at $0,15,30,45,60$, and 90 minutes. $n=5-9$ per group. Results are expressed as mean blood glucose concentration $\pm \mathrm{SEM}$; ${ }^{*} P<0.05$ vs. control mice; $\# P<0.05$ vs. Het $K O$ mice assessed by repeated-measures ANOVA.

by the fact that blood glucose failed to decrease after insulin injection ( $0.75 \mathrm{U} / \mathrm{kg}$ body weight), whereas in control littermates, glucose decreased about 40\% (Figure 3B). Het KO mice showed elevated initial glucose level (4 hours after food was removed) and an intermediate response to insulin (Figure 3B).

To determine whether glucose intolerance and insulin resistance worsens with age, we measured glucose tolerance in 6-month-old AMG4KO mice and compared the results to those from mice lacking GLUT4 in either tissue (Figure 4A). Fasting glucose was elevated and glucose tolerance was impaired in all 3 lines of GLUT4-KO mice. Surprisingly, glucose intolerance was not more marked in AMG4KO mice than in either $A G 4 K O$ or $M G 4 K O$ mice (Figure 4A). However, $A M G 4 K O$ mice had a higher risk for insulin resistance than $M G 4 K O$ mice, as evidenced by higher insulin levels in the fed state (Figure 4B). Fed insulin was increased 2.3-fold in AG4KO mice (left panel), 1.7-fold in $M G 4 K O$ mice (middle panel), and 3.4-fold in $A M G 4 K O$ mice (right panel) compared with their respective controls. Furthermore, in control littermates of $A M G 4 K O, 55 \%$ of the insulin values were less than $120 \mathrm{pM}$, whereas in Het KO, AMG4KO, and $A G 4 K O$ mice, none of the values were below this level. A range of fed insulin levels was observed in each of the genotypes studied, possibly due to different genetic modifiers in these outbred popula-

\section{Figure 4}

Effect of GLUT4 KO in both adipose tissue and skeletal muscle compared with single-tissue GLUT4 knockout mice. (A) Glucose tolerance tests were performed in female AG4KO, MG4KO, AMG4KO, and Het $K O$ mice compared with control littermates at the age of 5-6 months. $n=9-17$ per group. (B) Fed serum insulin levels were measured from female AG4KO, MG4KO, AMG4KO, and Het KO mice compared with control littermates at 4-8 months of age. Bars indicate mean values. ${ }^{*} P<0.05$ vs. control littermates; ${ }^{P} P<0.05$ vs. MG4KO mice; $¥ P<0.05$ vs. AMG4KO mice. tions. However, these results suggest that the higher level of insulin sensitivity (lower plasma insulin levels) seen in half the control group was eliminated in Het KO, AMG4KO, and $A G 4 K O$ mice. In addition, $56 \%$ of $A M G 4 K O$ mice and $30 \%$ of $H e t K O$ mice had insulin values greater than $300 \mathrm{pM}$, whereas none of the control littermates had values this high. Thus, heterozygous or homozygous disruption of GLUT4 from muscle and adipose tissue simultaneously is associated with an increased risk for elevated insulin levels (Figure 4B) and insulin resistance (Figure 3B).

Fed insulin levels were 2.6-fold higher in $A M G 4 K O$ mice compared with $M G 4 K O$ mice (Figure 4B). Interestingly, insulin levels in $A M G 4 K O$ mice were not significantly higher than in $A G 4 K O$ mice, which underscores the importance of GLUT4 in adipocytes in the regulation of whole-body insulin sensitivity. In Het $\mathrm{KO}$ mice, mean fed insulin was not elevated at 2 months (not shown) but became elevated by 4-8 months (Figure 4B), which indicates an age-related effect on insulin resistance as is seen in humans with type 2 diabetes and in mice that are heterozygous for GLUT4 targeting in all tissues (13). Fasting insulin levels in AMG4KO mice were also elevated 2-fold compared with control mice (Table 1). For all metabolic studies (Figures 5-8), AMG4KO mice with insulin values spanning the range shown in Figure 4B were used.

We found no significant differences between groups in fed plasma levels of adipokines. Leptin levels were $6.35 \pm 1.88 \mathrm{ng} / \mathrm{ml}$ in controls and $9.76 \pm 3.10 \mathrm{ng} / \mathrm{ml}$ in $A M G 4 K O$ mice $(P=\mathrm{NS})$. When data were expressed per gram of body weight for each mouse, there were still no differences between genotypes. Total adiponectin was $8.93 \pm 1.61 \mu \mathrm{g} / \mathrm{ml}$ in controls and $8.90 \pm 1.37$ $\mu \mathrm{g} / \mathrm{ml}$ in $A M G 4 K O$ mice $(P=\mathrm{NS})$. The proportion of high-molecular-weight adiponectin was low and did not appear to differ between groups $(1.5 \% \pm 0.7 \%$ of total adiponectin for controls, $0.2 \% \pm 0.2 \%$ for $A M G 4 K O ; P=N S)$. Resistin levels were not statistically different but tended to be increased $25-30 \%$ in $A M G 4 K O$ females (control, $6.88 \pm 1.04 \mathrm{ng} / \mathrm{ml}$; $A M G 4 K O, 8.60 \pm 0.95 \mathrm{ng} / \mathrm{ml}$;
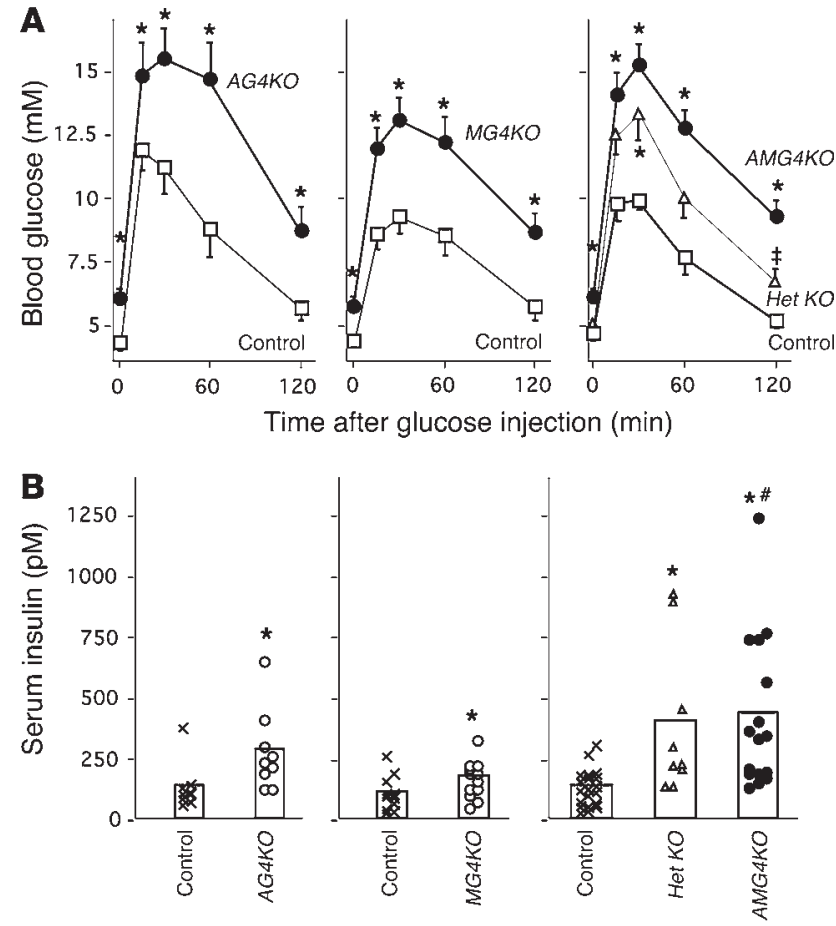
Table 1

Metabolic parameters before and during hyperinsulinemic-euglycemic clamp study in control and AMG4KO mice

\begin{tabular}{|c|c|c|c|c|c|c|}
\hline & \multirow[b]{2}{*}{$n$} & \multicolumn{3}{|c|}{ Basal period } & \multicolumn{2}{|c|}{ Clamp period } \\
\hline & & $\begin{array}{c}\text { Plasma } \\
\text { glucose }(\mathrm{mM})\end{array}$ & $\begin{array}{c}\text { Plasma } \\
\text { insulin (pM) }\end{array}$ & $\begin{array}{c}\text { HGP } \\
(\mu \mathrm{mol} / \mathrm{kg} / \mathrm{min})\end{array}$ & $\begin{array}{c}\text { Plasma } \\
\text { glucose }(\mathrm{mM})\end{array}$ & $\begin{array}{c}\text { Plasma } \\
\text { insulin (pM) }\end{array}$ \\
\hline Control mice & 8 & $7.0 \pm 0.7$ & $90 \pm 7$ & $107 \pm 17$ & $6.3 \pm 0.3$ & $666 \pm 76$ \\
\hline AMG4KO mice & 8 & $9.9 \pm 0.9^{A}$ & $189 \pm 30^{B}$ & $84 \pm 4$ & $6.5 \pm 0.7$ & $720 \pm 48$ \\
\hline
\end{tabular}

Studies were carried out in female mice at 5 months of age after an overnight fast. HGP, hepatic glucose production. ${ }^{A} P<0.01$ vs. control mice; ${ }^{B} P<0.05$ vs. control mice.

$50 \%$ and $120 \%$ higher, respectively, in liver of AMG4KO compared with controls (Figure 6B). Under non-clamp conditions as well, liver PEPCK and G6Pase mRNA levels tended to be higher in $A M G 4 K O$ mice than in controls (data not shown).

Impact of impaired muscle and adipose tissue glucose utilization on energy metabolism. We expected AMG4KO mice to be more severely diabetic since glucose uptake into muscle and adipose

$P=$ NS). It appears that $A M G 4 K O$ mice may have a higher risk for elevated plasma resistin levels. However, fed insulin levels did not correlate with resistin levels. There were no differences in levels of plasminogen activator inhibitor-1 (PAI-1) or adipose-secreted cytokines, IL-1 $\beta$, IL-6, IL-8, TNF- $\alpha$, or monocyte chemoattractant protein-1 (MCP-1).

Impact of decreasing GLUT4 in adipose tissue and muscle on in vivo glucose metabolism. To gain insight into glucose metabolism in different tissues, we performed 2-hour hyperinsulinemic-euglycemic clamp studies in awake 5-month-old female mice. Basal plasma glucose concentration was increased by approximately $40 \%$ in AMG4KO mice compared with control littermates $(P<0.01$; Table 1). During the clamp, plasma insulin concentrations were raised to approximately $700 \mathrm{pM}$, and plasma glucose was clamped at 6.3-6.5 $\mathrm{mM}$. The rate of glucose infusion needed to maintain euglycemia increased rapidly in the control mice. In contrast, glucose infusion rate (GINF) in response to insulin was markedly reduced (by approximately $75 \%$ ) in $A M G 4 K O$ mice (Figure $5 \mathrm{~A}$ ). Insulin-stimulated whole-body glucose uptake and whole-body glycolysis were reduced by $60-65 \%$ in $A M G 4 K O$ mice, and glycogen synthesis tended to be reduced (Figure 5A).

To determine which tissues are responsible for the decrease in insulin-stimulated whole-body glucose utilization in $A M G 4 K O$ mice, we measured the rate of insulin-stimulated 2-deoxyglucose (2-DG) uptake in individual tissues during the last 40 minutes of the clamp (Figure 5B). There was no difference in 2-DG uptake rate in cerebellum between $A M G 4 K O$ and control mice. In extensor digitorum longus and gastrocnemius muscle, 2-DG uptake rates were markedly depressed by $85-90 \%$ in AMG4KO mice. In soleus muscle and heart, insulin-stimulated 2-DG uptake in $A M G 4 K O$ was decreased by $75 \%$. In $A M G 4 K O$ WAT and BAT, 2-DG uptake was decreased approximately $65 \%$ and $50 \%$, respectively (Figure $5 \mathrm{~B}$ ). The induction of GLUT1 expression in BAT and heart (Figure 1) may have contributed to the smaller defects in these tissues.

Impact of impaired muscle and adipose tissue glucose utilization on hepatic insulin sensitivity. Hepatic glucose production (HGP) was normal in the basal state (Table 1), but the ability of insulin to suppress HGP was impaired by $50 \%$ in $A M G 4 K O$ mice (Figure $6 \mathrm{~A}$ ). To understand the mechanism, we measured the expression of the gluconeogenic enzymes, phosphoenolpyruvate carboxykinase (PEPCK) and glucose-6-phosphatase (G6Pase), in liver samples after the insulin clamp. These enzymes are important determinants of HGP. Their expression is increased by fasting and is rapidly inhibited at the transcriptional level by insulin. Northern blot analysis revealed that after insulin infusion in fasted mice, PEPCK and G6Pase mRNA levels normalized to $\beta$-actin mRNA levels were tissue was so markedly impaired (Figure 5B). We wanted to understand more about fuel utilization in these mice, and we hypothesized that they might have increased lipid oxidation, which could enhance their insulin sensitivity (14). To test this, we performed indirect calorimetry and calculated the respiratory quotient (RQ), which is a ratio of carbohydrate oxidation to lipid oxidation. During the light period, the RQ was lower in $A M G 4 K O$ mice compared with control littermates (Figure 7A). This indicates that AMG4KO mice have relatively lower carbohydrate utilization and higher fat utilization than control littermates, even though control mice are also primarily metabolizing lipid stores due to low levels of food intake during the light period. During the dark period, the RQ rose from 0.72 to
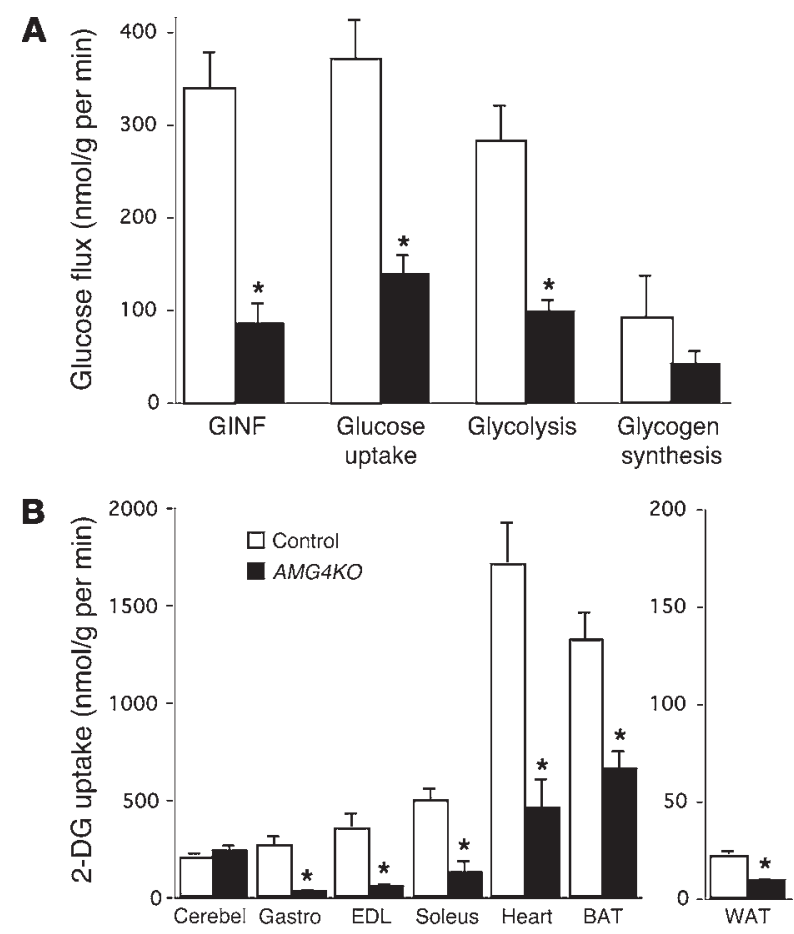

Figure 5

In vivo glucose metabolism during hyperinsulinemic-euglycemic clamp studies in awake female AMG4KO mice at 6 months of age. (A) GINF, insulin-stimulated whole-body glucose uptake, glycolysis, and glycogen synthesis in control (white bars) and AMG4KO (black bars) mice. (B) Insulin-stimulated glucose uptake in cerebellum (Cerebel), gastrocnemius, extensor digitorum longus (EDL), soleus, heart, BAT, and WAT of control and $A M G 4 K O$ mice in vivo. Values are means \pm SEM; ${ }^{\star} P<0.05$ vs. control littermates. $n=8$ per group. 

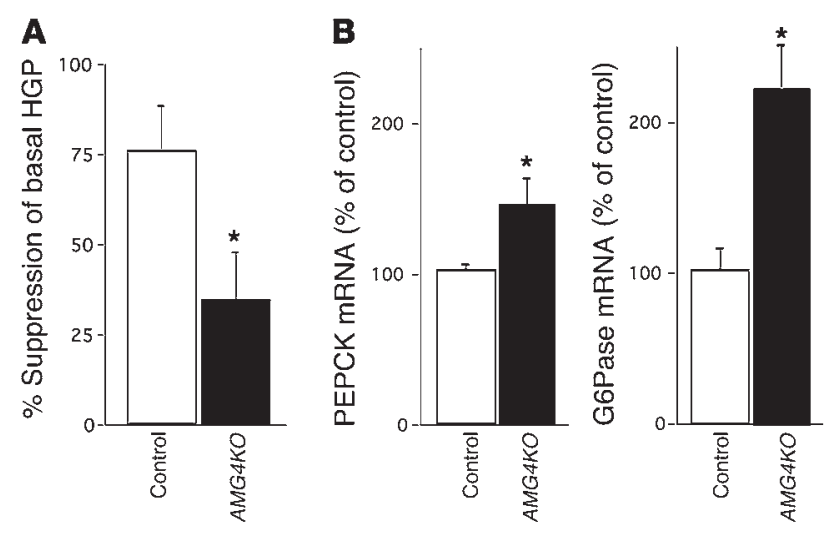

Figure 6

Insulin action in liver during hyperinsulinemic-euglycemic clamp study in awake female AMG4KO mice. (A) Percent suppression of basal hepatic glucose production in control and AMG4KO mice. (B) Insulin inhibition of PEPCK and G6Pase gene expression in liver of control and $A M G 4 K O$ mice. RNA was prepared from liver after the hyperinsulinemic-euglycemic clamp study and was subjected to Northern blot analysis. PEPCK and G6Pase mRNA levels were normalized to $\beta$-actin mRNA levels and expressed as $\%$ of control levels. ${ }^{\star} P<0.05$ vs. control littermates. $n=6-8$ per group.

0.92 in control mice as they ate chow that is high in carbohydrate. In AMG4KO mice, the RQ rose from 0.66 to only 0.76 , which reflected their continued dependence on lipid metabolism, most likely due to the impairment of glucose uptake in muscle and adipocytes.

To examine whether the AMG4KO mice could clear lipids more efficiently, we administered exogenous triglycerides intragastrically 4 hours after food removal and monitored serum triglyceride and FFA concentrations. Initial triglyceride and FFA levels were normal in AMG4KO mice (Figure 7B). Serum triglyceride levels in mice of both genotypes gavaged with saline decreased by more than $90 \%$ during the first 2 hours (Figure 7B, squares). In contrast, the serum triglyceride levels in mice of both genotypes gavaged with lipids increased to $240 \mathrm{mg} / \mathrm{dl}$ over the first 2 hours. After that, the clearance of triglycerides in $A M G 4 K O$ mice was faster than in control littermates (Figure 7B, left panel). Similarly, serum FFA levels were normal in $A M G 4 K O$ mice before the gavage and rose comparably in control and $A M G 4 K O$ mice gavaged with lipid (Figure 7B, right panel). However, the clearance of FFA after 2 hours was faster in AMG4KO mice than in the control littermates. The comparable rise in triglycerides and FFA in the 2 genotypes suggests that the absorption of lipid from the gut was normal. There was no excess lipid in the feces of the AMG4KO

\section{Figure 7}

Energy metabolism in AMG4KO mice. (A) Twenty-four-hour respiratory quotient was measured by indirect calorimetry in ad libitum-fed female mice. Light period data were collected for 5 hours starting from 13:00, and dark period data were collected for 5 hours starting from 22:00. ${ }^{*} P<0.05$ vs. same period in control littermates. (B) Serum triglyceride and FFA levels during a fat-loading test. After a 4-hour fast, control (open symbols) and AMG4KO (closed symbols) mice were gavaged with olive oil (16.7 $\mu \mathrm{l} / \mathrm{g}$ body weight; circles) or saline (rectangles). Before and after fat loading, blood was collected serially, and serum triglyceride and FFA levels were measured. ${ }^{*} P<0.05$ vs. control littermates given lipid. $n=6$ per group given lipids, $n=1$ per group given saline. mice. Thus, the enhanced lipid clearance in $A M G 4 K O$ mice reflects an increased ability to metabolize triglycerides.

Hepatic lipid metabolism and glucose flux. There was no significant difference in serum triglyceride concentrations in the fed state between AMG4KO and control mice $(76 \pm 11 \mathrm{mg} / \mathrm{dl}$ vs. $104 \pm 14 \mathrm{mg} / \mathrm{dl}$, respectively; $P=0.79$ ). To determine whether there was an enhanced supply and production of lipids, we injected Triton WR1339 in awake mice to block serum triglyceride clearance and measured the increase in serum triglycerides. The injection of Triton WR1339 resulted in a linear increase in serum triglyceride concentrations for 4 hours after injection (Figure 8A). Linear regression analysis of the increase in serum triglyceride concentrations revealed that the rate of hepatic triglyceride production in AMG4KO mice was increased 1.5 -fold compared with control littermates $(6.3 \pm 0.8 \mathrm{mg} / \mathrm{dl}$ vs. $4.3 \pm 0.5 \mathrm{mg} / \mathrm{dl}$ per hour; $P<0.05$ ), which suggests increased hepatic VLDL synthesis in $A M G 4 K O$ mice.

To investigate whether increased VLDL-triglyceride secretion could be caused by increased de novo lipogenesis, we injected mice with both tritiated water and $\left[\mathrm{U}-{ }^{14} \mathrm{C}\right]$-glucose and assessed de novo lipogenesis. The total rate of hepatic fatty acid synthesis (measured by ${ }^{3} \mathrm{H}_{2} \mathrm{O}$ incorporation into fatty acids) was 2 -fold higher in AMG4KO mice compared with control littermates $(P<0.05)$ (Figure $8 \mathrm{~B}$ left panel). The incorporation of $\left[\mathrm{U}-{ }^{14} \mathrm{C}\right]$-glucose into de novo fatty acids was increased more than 3-fold in $A M G 4 K O$ mice compared with control littermates $(P<0.05)$ (Figure $8 \mathrm{~B}$, right panel).

Expression of the key lipogenic enzyme, acetyl-CoA carboxylase (ACC), was increased 1.6-fold in liver of AMG4KO mice compared with control littermates (Figure 8C). This effect was specific to liver, since ACC levels were decreased in WAT and not changed in BAT, muscle, or heart of $A M G 4 K O$ mice (Figure 8C). SREBP-1c is a membrane-bound transcription factor that regulates enzymes responsible for fatty acid metabolism (15). SREBP-1c mRNA levels normalized to $\beta$-actin mRNA were increased by 1.9 -fold in $A M G 4 K O$ mice (Figure 8D). In spite of these changes, hepatic
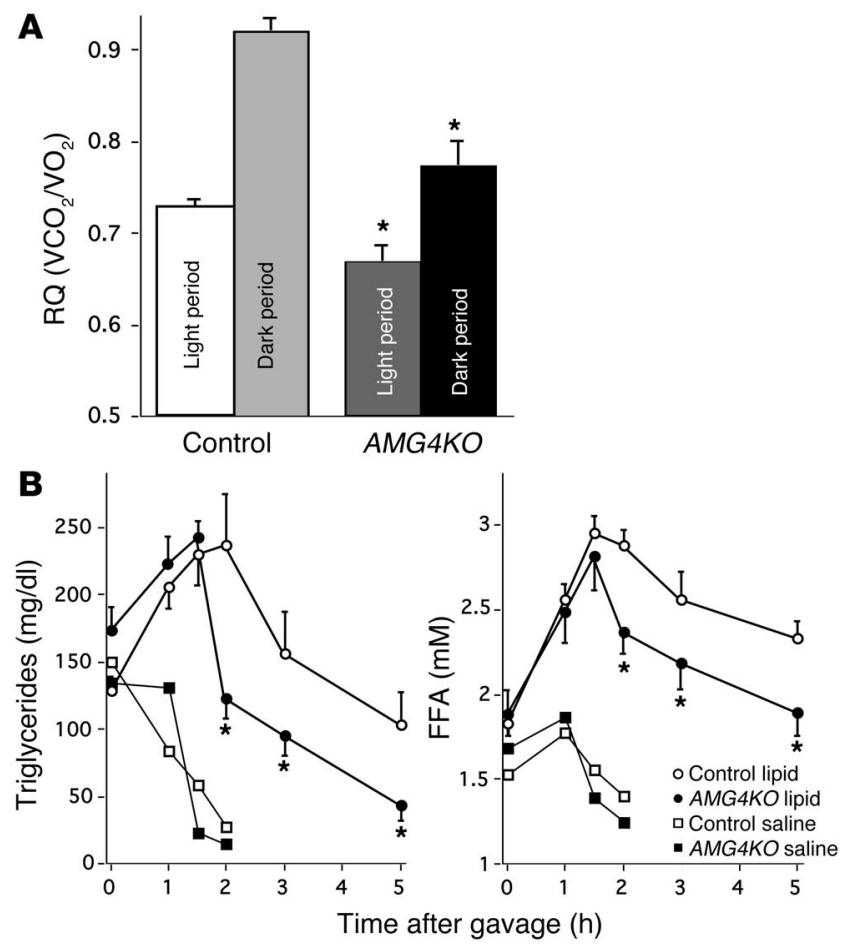

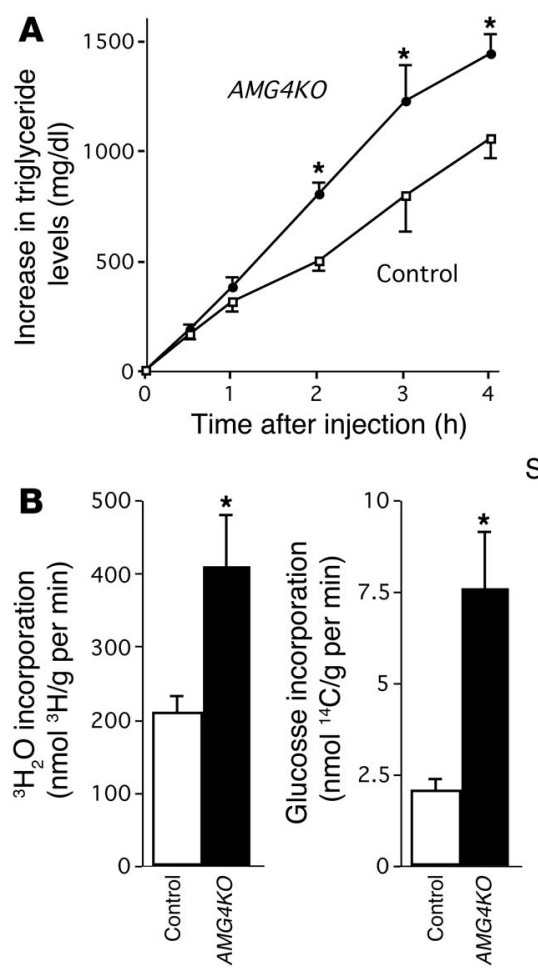

D
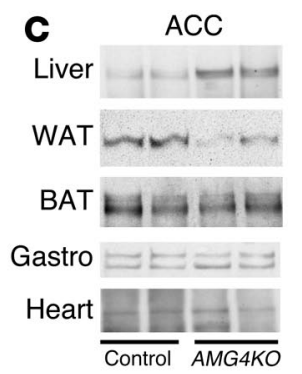

D

SREBP-1C

Actin

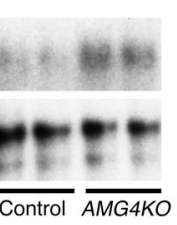

$\mathbf{E}$

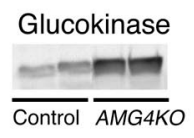

triglyceride content did not differ between $A M G 4 K O$ and control littermates $(18.1 \pm 1.8 \mathrm{mg} / \mathrm{g}$ vs. $16.5 \pm 2.1 \mathrm{mg} / \mathrm{g}$ of liver, respectively). Muscle triglyceride content was also not altered in $A M G 4 K O$ compared with control mice $(13 \pm 4 \mathrm{mg} / \mathrm{g}$ in $A M G 4 K O$ vs. $16 \pm 3$ $\mathrm{mg} / \mathrm{g}$ of gastrocnemius in control). Thus, increased production of triglycerides is offset by increased utilization, so there is no increase in tissue storage of triglycerides. The in vivo lipogenesis data (Figure 8B) demonstrate that part of the adaptation to the impairment of glucose transport in muscle and adipose tissue is increased glucose incorporation into fatty acids in the liver.

Glucokinase is a key enzyme in determining glucose utilization by the liver, and in some aspects it is a marker for glucose uptake in the liver. Glucokinase protein levels were increased 2.3-fold in the liver of $A M G 4 K O$ mice (Figure $8 \mathrm{E}$ ), which suggests increased glucose uptake. This is confirmed by the measured increase in glucose incorporation into lipids in the liver of $A M G 4 K O$ mice compared with controls (Figure $8 \mathrm{~B}$ ). Thus, the lack of a more marked elevation of blood glucose levels in $A M G 4 K O$ mice could be because the liver is compensating by taking up more glucose.

\section{Discussion}

In humans with obesity and type 2 diabetes and in rodent models of obesity and diabetes, insulin-stimulated glucose transport is impaired in both adipose tissue and skeletal muscle (2). There is still controversy about the role of defects in glucose uptake in peripheral tissues that occur early in the pathogenesis of type 2 diabetes compared with defects in insulin action on the liver or insulin secretory defects. To address the importance of insulin-stimulated glucose transport and to understand the adaptations that occur when glucose utilization is impaired in muscle and adipose tissue, we generated and characterized mice lacking GLUT4 in both of these tissues. Our results in this animal model demonstrate that when glucose utilization in muscle and adipose tissue is impaired,

\section{Figure 8}

VLDL-triglycerides production and hepatic de novo lipogenesis in female AMG4KO mice. (A) Determination of VLDL-triglyceride production rates. After a 4-hour fast Triton WR 1339 was injected i.v. and serum samples were taken for a period of $4 \mathrm{~h}$ and assayed for triglycerides in $A M G 4 K O$ (filled circles) and control (open squares) littermates. ${ }^{*} P<0.05$ vs. control littermates. $n=6-7$ per genotype. (B) Rates of ${ }^{3} \mathrm{H}_{2} \mathrm{O}$ (left panel) and ${ }^{14} \mathrm{C}$-glucose (right panel) incorporation into liver fatty acids in fed state. $n=7-8$ per genotype. (C) ACC expression in female control and $A M G 4 K O$ mice after the hyperinsulinemic-euglycemic clamp study. Tissue extracts from liver, WAT, BAT, gastrocnemius, and heart were prepared and immunoblotted with peroxidase-labeled streptavidin to detect the relative content of ACC isoforms. (D) RNase protection assay for SREBP-1c mRNA in livers of control and AMG4KO mice. Total RNA $(30 \mu \mathrm{g})$ isolated from liver was subjected to RNase protection assay. The data were normalized relative to $\beta$-actin mRNA. (E) A representative Western blot analysis of glucokinase in liver of female control and AMG4KO mice. $n=8$ per genotype.

as it is in type 2 diabetes, muscle adapts by shifting to utilization of lipid fuels, whereas liver appears to increase glucose uptake, glucose incorporation into fatty acids, and triglyceride production. These metabolic adaptations seem to result in protection from developing an even greater degree of insulin resistance/hyperinsulinemia or more marked hyperglycemia under ambient conditions. Since insulin-resistant humans appear to lack such "metabolic flexibility" (7), this model may shed light on how this lack of adaptability contributes to the pathogenesis of type 2 diabetes.

Is GLUT4 required for growth, normal adipose mass, or normal longevity? Unlike GLUT4-null mice, which display growth retardation and severely reduced adipose tissue deposits (12), AMG4KO mice have normal growth and normal body composition (Figure 2). The reason for this difference is unclear but might be due to a possible role for GLUT4 in certain regions of the brain, kidney, or other tissues $(10,11)$ in the regulation of growth and adiposity. In fact, it has been suggested that the presence of GLUT4 in murine brain, with highest concentrations during the suckling phase, may have a unique role in augmenting substrate delivery under conditions of increased demand during development (16). Thus, GLUT4 may play a role in the nervous regulation of adipose tissue development and metabolism. To address these issues, generation of brain-specific GLUT4-knockout mice would be informative. GLUT4-null mice also have decreased longevity, which is evident by 6 months of age (12). In contrast, $A M G 4 K O$ mice have a normal life span (at least until 1.5 years of age) in spite of cardiomegaly.

Does impaired glucose utilization in muscle and adipose tissue due to GLUT4 disruption cause severe insulin resistance or diabetes? AMG4KO mice showed insulin resistance and glucose intolerance as early as 2 months of age (Figure 3), but the degree of glucose intolerance in $A M G 4 K O$ mice was not greater than that observed in $M G 4 K O$ or $A G 4 K O$ mice (Figure 4A). This may be because mice lacking GLUT4 in either tissue secondarily develop insulin resistance in the other insulin target tissues, so they also have defects in glucose uptake in both tissues. However, $A M G 4 K O$ mice are at risk for a greater degree of insulin resistance than $M G 4 K O$ or $A G 4 K O$ mice (Figure 4B). Ambient insulin levels in $A M G 4 K O$ mice were elevated 2.6-fold compared with $M G 4 K O$ mice (Figure 4B). Even though the average level of hyperinsulinemia in the $A M G 4 K O$ mice was not statistically significantly higher than in the $A G 4 K O$ mice, the upper range of insulinemia values in the AMG4KO mice was much higher (Figure 4B). The heterogeneity is characteris- 
tic of outbred genetic lines and reflects genetic modifiers that impact on the severity of the phenotype. Since insulin-stimulated glucose uptake is markedly impaired in vivo in both muscle and adipose tissue in $A M G 4 K O$ mice (Figure 5B), it may be surprising that $A M G 4 K O$ mice are not more hyperglycemic under ambient conditions. While GLUT1 expression is increased in heart and BAT of these mice (Figure 1), it is not upregulated in skeletal muscle or WAT, and so this cannot compensate for the lack of GLUT4 in terms of whole-body glucose homeostasis.

Does impaired muscle and adipose tissue glucose utilization cause hepatic insulin resistance? The ability of insulin to suppress HGP was significantly reduced in $A M G 4 K O$ mice (Figure 6A), consistent with results in $M G 4 K O$ and in $A G 4 K O$ mice. These data confirm that hepatic insulin resistance can develop secondarily to impaired glucose utilization in muscle and/or adipose tissue. Here we show that the mechanism appears to involve resistance to insulin's ability to suppress expression of PEPCK and G6Pase (Figure 6B), the ratelimiting enzymes for gluconeogenesis. Interestingly, in GLUT4 $4^{+-}$ mice, the ability of insulin to inhibit HGP was normal (17), despite impairment in whole-body insulin-mediated glucose disposal. This difference may be due to the fact that in the GLUT4 ${ }^{+-}$mice, compared with $A M G 4 K O$ mice, peripheral glucose uptake is reduced by only $50 \%$ and glycemia is normal. Another possible explanation is that the supraphysiological concentration of insulin $(18 \mathrm{mU} / \mathrm{kg} /$ min) used in ref. 17 might be sufficient to completely suppress HGP in GLUT4 ${ }^{+/-}$mice. In the present study, we used a lower level of insulin $(2.5 \mathrm{mU} / \mathrm{kg} / \mathrm{min})$, which inhibited HGP by $75 \%$ in the control mice but by only $35 \%$ in the AMG4KO mice (Figure $6 \mathrm{~A}$ ).

Does impaired muscle and adipose tissue glucose utilization alter energy metabolism? The fact that AMG4KO mice are not more severely diabetic could possibly be due to compensation by other tissues/ organs that might use more glucose. We hypothesized that in AMG4KO mice, the liver utilizes more glucose and produces more lipids that could be used as fuel substrates by muscle and adipose tissue. The impairment of glucose uptake in muscle and adipose tissue of the $A M G 4 K O$ mice could induce a metabolic adaptation whereby these tissues use a greater proportion of fatty acids versus glucose compared with control mice.

Indeed, we observed a markedly lower RQ in AMG4KO than in control mice (Figure 7A), which reflects a shift in fuel utilization from glucose to fatty acids. Of note, the difference in the respiratory quotient was more marked during the dark period, when the animals ate, eliciting insulin secretion and glucose utilization. This is consistent with the expected metabolic effect of a lack of insulininduced (GLUT4-mediated) glucose uptake by peripheral tissues.

In accordance with the apparent greater reliance of $A M G 4 K O$ mice on lipids, these mice displayed an increased hepatic de novo lipogenic activity (Figure 8B) as well as a higher rate of clearance of serum triglycerides and free fatty acids (Figure 7B). These results suggest that impaired glucose utilization by muscle and adipose tissue can trigger metabolic adaptations that allow a greater production of fatty acids from glucose by the liver and a greater utilization of fatty acids as fuel substrate by peripheral tissues. This mechanism might explain the fact that in insulin-resistant humans, impaired glucose uptake by peripheral tissues in the postprandial period is associated with a higher rate of lipid oxidation (7).

Why didn't AMG4KO mice develop more severe diabetes? We investigated the mechanisms that allow the $A M G 4 K O$ mice to utilize glucose by routes other than glucose transport in muscle and adipose tissue. In addition to releasing glucose, the liver takes up and metab- olizes glucose. Phosphorylation of glucose by glucokinase is the rate-limiting step in hepatic glucose metabolism. When activated, it increases glucose uptake and storage as glycogen and limits glucose export through gluconeogenesis. Alterations in glucokinase expression or activity have major effects on glucose homeostasis, as evidenced by the fact that liver-specific glucokinase-KO mice exhibit mild hyperglycemia (18) and rats overexpressing glucokinase in the liver have reduced blood glucose and insulin levels, which suggests an increase in hepatic glucose uptake (19). Furthermore, allosteric activators of glucokinase lower blood glucose in diabetic rodents by altering hepatic glucose metabolism and glucose-stimulated insulin secretion (20). In type 2 diabetic patients, the ability of insulin to increase liver glucokinase activity is defective $(21,22)$, as is liver glucose uptake (23).

In $A M G 4 K O$ mice, glucokinase expression in the liver is increased (Figure 8E), which indicates increased hepatic glucose uptake. As evidence of the latter, direct measurement of glucose incorporation into lipids in the liver and VLDL-triglyceride production (Figure 8, A and B) revealed marked increases in AMG4KO mice. Thus, the upregulation of hepatic glucokinase expression in these mice might represent an adaptive mechanism by the liver to take up more glucose under conditions of impaired glucose utilization by muscle and adipose tissue. The fact that patients with type 2 diabetes do not display the same metabolic adaptation may contribute to their hyperglycemia, especially since the relative contribution of splanchnic glucose uptake to overall glucose disposal is greater in diabetic subjects (21-23).

In $A M G 4 K O$ mice, increased hepatic de novo lipogenesis from glucose and increased VLDL-triglyceride secretion by the liver might ensure an adequate supply of fuel substrate for the muscle, heart, and adipose tissue. The fact that $A M G 4 K O$ mice preferentially use fatty acids because glucose uptake in muscle and adipose tissue is blocked might explain why they do not show elevations in circulating triglyceride and free fatty acid levels, unlike rats overexpressing glucokinase in the liver (19).

SREBP-1c regulates the expression of genes involved in fatty acid metabolism (24), and ACC, which catalyzes the biosynthesis of malonyl-CoA from acetyl-CoA, is a key lipogenic enzyme. The elevated expression of SREBP-1c and ACC in the liver of AMG4KO mice is likely to play a role in the increased de novo lipogenic activity. De novo synthesis of fatty acids by the liver makes a minor contribution to overall triglyceride secretion but has been suggested to be important for regulation of VLDL production $(25,26)$. Hepatic triglyceride stores (an important source of lipids for VLDL assembly; ref. 27) are normal in $A M G 4 K O$ mice, probably because increased de novo lipogenesis is balanced by VLDL secretion.

Summary. The suggested compensatory increase in hepatic glucose utilization in AMG4KO mice represents a metabolic link among skeletal muscle, adipose tissue, and liver. This adaptation could play a key role in determining the severity of whole-body insulin resistance due to impaired glucose uptake, e.g., at the level of GLUT4, and it may prevent the development of even more severe insulin resistance and more overt diabetes in $A M G 4 K O$ mice. Our results showing greater hepatic de novo lipogenesis and production of VLDL-triglycerides in AMG4KO mice suggest that dyslipidemia in obese, insulin-resistant humans could be due to an adaptive mechanism by the liver in response to insulin resistance in muscle and adipose tissue. In contrast to our mouse model, however, most insulin-resistant humans lack the adaptive capacity to increase utilization of these lipids (7). The dynamics 
in the AMG4KO mice indicate that this could be a factor in the severity of the diabetic phenotype.

AMG4KO mice demonstrate that perturbations of glucose (or fatty acid) utilization in one tissue will affect energy metabolism in other tissues. Pathologic consequences of impaired insulininduced glucose utilization might depend, in part, on differences in the capability of peripheral tissues to adapt to alterations in fuel substrate availability. It is likely that in humans, genetic determinants affecting these adaptive processes play a role in the development and progression of insulin resistance and diabetes. Recent data showing defective expression of genes involved in mitochondrial oxidative phosphorylation in humans with insulin resistance and type 2 diabetes $(28,29)$ support this notion.

\section{Methods}

Animals. GLUT4 loxP mice derived from C57BL/6J were created by homologous recombination using a GLUT4 gene targeting vector with loxP sites flanking exon 10, as previously described (30). FVB/N mice carrying the aP2-Cre transgene were made by cloning a 1.4-kb SacI/SalI complementary DNA fragment encoding Cre recombinase immediately downstream of the 5.4-kb promoter/enhancer of fatty acid-binding protein aP2 (8). Transgenic mice with $M C K$-driven expression of Cre recombinase were produced by subcloning the Cre cDNA containing a nuclear localization signal in place of the translation initiation site of the MCK gene (31). Animals were housed in a 12-hour dark-light cycle and fed standard chow ad libitum. All aspects of animal care and experimentation were approved by the Institutional Animal Care and Use Committee of the Beth Israel Deaconess Medical Center and Harvard Medical School and Brandeis University. In the $A M G 4 K O$ group, we studied all animals that showed a greater than $70 \%$ decrease in GLUT4 protein content in WAT and 90\% in skeletal muscle.

Immunoblotting. Tissue detergent extracts were prepared by homogenization in lysis buffer (20 mM Tris HCl, pH7.4, 5 mM EDTA, 1\% NP-40, 10 $\mathrm{mM} \mathrm{Na}_{4} \mathrm{P}_{2} \mathrm{O}_{7}, 100 \mathrm{mM} \mathrm{NaF}, 2 \mathrm{mM} \mathrm{Na}_{3} V_{4}, 1 \mathrm{mM}$ PMSF, $10 \mu \mathrm{g} / \mathrm{ml}$ aprotinin, and $10 \mu \mathrm{g} / \mathrm{ml}$ leupeptin) using a polytron at half-maximum speed for 1 minute on ice. GLUT4 and GLUT1 protein levels were determined using polyclonal, carboxyterminal antibodies (from H. Haspel, Charles River Laboratory, Wilmington, Massachusetts, USA; and B. Thorens, Institute of Pharmacology and Toxicology, Lausanne, Switzerland; respectively), as previously described (30). Acetyl-CoA carboxylase protein levels were determined using peroxidase-labeled streptavidin (Amersham Pharmacia Biotech Inc.). Glucokinase protein levels were determined using sheep anti-glutathione-S-transferase-glucokinase fusion protein antibody (gift from M.A. Magnuson, Vanderbilt University, Nashville, Tennessee, USA).

Body composition. A PIXIMUS Densitometer (LUNAR Corp.) was used for the measurement of body composition in anesthetized mice (dual-energy $\mathrm{x}$-ray absorptiometry analysis) (32).

Glucose and insulin tolerance tests. Glucose tolerance tests were performed by intraperitoneal injection of glucose ( $1 \mathrm{~g}$ D-glucose/ $\mathrm{kg}$ body weight) after an overnight fast. Insulin tolerance tests were performed by intraperitoneal injection of human regular insulin $(0.75 \mathrm{U}$ insulin $/ \mathrm{kg}$ body weight; Eli Lilly and Co.) 4 hours after removal of food. Blood glucose was determined with a One Touch Profile glucometer (Lifescan Inc.).

Hyperinsulinemic-englycemic clamp with 2-DG uptake in vivo. At least 4 days before experiments, mice were anesthetized with $90 \mathrm{mg} / \mathrm{kg}$ body weight ketamine (Sigma-Aldrich) and $10 \mathrm{mg} / \mathrm{kg}$ body weight xylazine (SigmaAldrich), and an indwelling catheter (BD) was inserted in the right internal jugular vein. After an overnight fast, a 120-minute hyperinsulinemiceuglycemic clamp was conducted in awake mice, as previously described (33). A prime-continuous infusion of human insulin (Eli Lilly and Co.) at a rate of $15 \mathrm{pmol} / \mathrm{kg} / \mathrm{min}$ raised plasma insulin to approximately
660-720 pM (Table 1), and 40\% glucose was infused at variable rates and periodically adjusted to clamp the plasma glucose levels at approximately $6.5 \mathrm{mM}$ using microdialysis pumps (CMA/Microdialysis). $\left[3-{ }^{3} \mathrm{H}\right]$ glucose (10 $\mu \mathrm{Ci}$ bolus, followed by $0.1 \mu \mathrm{Ci} \mathrm{min}^{-1}$; NEN Life Sciences) was infused throughout the clamp to estimate insulin-stimulated whole-body glucose flux. Insulin-stimulated glucose uptake in individual tissues was estimated by administration of a bolus $(10 \mu \mathrm{Ci})$ of 2 -deoxy-D-[1-14 C]glucose $\left(2-\left[{ }^{14} \mathrm{C}\right] \mathrm{DG}\right.$; Amersham Pharmacia Biotech Inc.). At the end of the clamp study, mice were euthanized with ketamine and xylazine injection, and tissues were frozen in liquid nitrogen for subsequent analysis. Plasma samples were analyzed for insulin and for glucose concentration by a glucose oxidation method (Sigma-Aldrich) and for $\left[{ }^{3} \mathrm{H}\right]$ glucose, $2-\left[{ }^{14} \mathrm{C}\right] \mathrm{DG}$, and ${ }^{3} \mathrm{H}_{2} \mathrm{O}$ concentrations as previously described (33). Rates of basal glucose turnover and whole-body glucose uptake were determined as the ratio of the $\left[{ }^{3} \mathrm{H}\right]$ GINF (disintegrations per kilogram per minute) to the specific activity of plasma glucose (disintegration per micromole) at the end of the basal period and during the final 40 minutes of clamp period, respectively. Hepatic glucose production during the hyperinsulinemiceuglycemic clamps was determined by subtracting the rate of glucose infusion from the rate of whole-body glucose uptake. Whole-body glycolysis was calculated from the rate of increase in plasma ${ }^{3} \mathrm{H}_{2} \mathrm{O}$ concentration, determined by linear regression of the measurements at 80,90 , 100,110 , and 120 minutes. We estimated whole-body glycogen synthesis by subtracting the rate of whole-body glycolysis from the rate of wholebody glucose uptake, assuming that glycolysis and glycogen synthesis account for the majority of insulin-stimulated glucose uptake (34). Glucose uptake in individual tissues was calculated from the plasma $2-\left[{ }^{14} \mathrm{C}\right] \mathrm{DG}$ profile, which was fitted with a double exponential curve using Origin (OriginLab Corp.) and tissue 2-DG-6-phosphate content.

RNA preparation, Northern blot analysis, and RNase protection assay. Total RNA from liver was extracted using Trizol reagent (Life Technologies). For Northern blot analysis, equal aliquots of total RNA were subjected to electrophoresis in a $1.2 \%$ agarose gel and transferred to nylon membrane (Osmonics) for hybridization. The filters were hybridized to ${ }^{32} \mathrm{P}$-labeled PEPCK (a gift from R. W. Hanson, Case Western Reserve University School of Medicine, Cleveland, Ohio, USA), G6Pase (a gift from L. Rossetti, Albert Einstein College of Medicine, Bronx, New York, USA), and $\beta$-actin (BD Biosciences - Clontech) cDNA.

For RNase protection assay, aliquots of total RNA $(30 \mu \mathrm{g})$ were hybridized with ${ }^{32} \mathrm{P}-$ labeled SREBP-1c riboprobe (a gift from I. Shimomura, Osaka University, Osaka, Japan) and $\beta$-actin using the HybSpeed RPA kit (Ambion Inc.). The protected bands were separated by polyacrylamide gel electrophoresis, exposed to film, and quantified using Storm (Amersham Pharmacia Biotech Inc.).

Indirect calorimetry. In vivo indirect calorimetry was performed in metabolic chambers using a 4-chamber Oxymax system (Columbus Instruments). To calculate oxygen consumption $\left(\mathrm{VO}_{2}\right)$, carbon dioxide production $\left(\mathrm{VCO}_{2}\right.$ ), and RQ (ratio of $\mathrm{VCO}_{2}$ to $\mathrm{VO}_{2}$ ), gas concentrations were monitored at the inlet and outlet of the sealed chambers.

Fat loading test. Oral olive oil tolerance tests were conducted as described previously (35). During a 4-hour period, mice were not given food but had ad libitum access to water. Olive oil $(16.7 \mu \mathrm{l} / \mathrm{g}$ body) was introduced intragastrically using gavage. After fat loading, approximately $50 \mu \mathrm{l}$ of blood was collected serially at $0,0.5,1,2,3$, and 5 hours, and serum triglyceride levels and FFA concentrations were measured.

In vivo VLDL-triglyceride production using Triton WR1339. Mice were injected intravenously with $600 \mathrm{mg}$ of Triton WR1339 (Sigma-Aldrich) per kilogram body weight as a $15 \%$ solution in $0.9 \% \mathrm{NaCl} 4$ hours after food was removed. Previous studies have shown that plasma VLDL clearance is virtually completely inhibited under these conditions (36). Blood samples were 
taken at $0,0.5,1,2,3$, and 4 hours after Triton WR1339 injection, and serum triglycerides were measured.

Metabolite measurements. Ambient insulin concentrations and plasma human insulin concentrations during the hyperinsulinemic study were determined by enzyme-linked immunosorbent assay using a rat insulin kit with mouse insulin standard (Crystal Chem. Inc.) and a human insulin ELISA kit (Linco Research Inc.), respectively. Serum triglyceride and FFA concentrations were measured using a colorimetric kit (Sigma-Aldrich) and a NEFA C kit (Wako), respectively. Fed plasma levels of leptin, TNFo, IL-1 $\beta$, IL-6, IL-8, PAI-1, MCP-1, and resistin were measured using Mouse Serum Adipokine Lincoplex kit (Linco Research Inc.). Fed plasma total adiponectin levels were determined using a mouse kit from Linco Research Inc., and the proportions of high-molecular-weight adiponectin complexes were measured by Philip Scherer's laboratory as previous described (37). Tissue glycogen content was analyzed as follows: tissue samples were dissolved in $0.5 \mathrm{~N} \mathrm{KOH}$ at $95^{\circ} \mathrm{C}$. The samples were precipitated in ethanol at $-80^{\circ} \mathrm{C}$. Precipitated glycogen was hydrolyzed with aminoglucosidase (Sigma-Aldrich). The amount of released glucose was determined using Trinder glucose oxidase reagent (Sigma-Aldrich). Tissue triglyceride contents were analyzed as previous described (38).

In vivo lipogenesis. Mice in the fed state were injected intraperitoneally with $1 \mathrm{mCi}$ of ${ }^{3} \mathrm{H}_{2} \mathrm{O}$ (Sigma-Aldrich) and $10 \mu \mathrm{Ci}$ of $\left[\mathrm{U}-{ }^{14} \mathrm{C}\right.$-glucose (ICN Pharmaceuticals Inc.) and sacrificed 1 hour later using an overdose of ketamine and xylazine (160 and $24 \mathrm{mg} / \mathrm{kg}$ i.p., respectively). Samples of plasma were obtained serially at $5,10,30$, and 60 minutes after injection for measurement of the plasma glucose levels and the specific activity of plasma water and glucose. The liver was rapidly removed, frozen in liquid $\mathrm{N}_{2}$, and then homogenized with chloroform/methanol. ${ }^{3} \mathrm{H}$ and ${ }^{14} \mathrm{C}$ incorporation into saponified fatty acid extracted from tissues was measured (38). The rate of de novo lipogenesis was calculated as nanomoles of ${ }^{3} \mathrm{H}$ incorporated into lipid/gram tissue per minute. Glucose incorporation into fatty acids was calculated as nanomoles of ${ }^{14} \mathrm{C}$-glucose incorporated into fatty acids/gram tissue per minute, using ${ }^{14} \mathrm{C}$ counts in saponified fatty acids extracted from tissue and in plasma at each time point.

Statistical analysis. Data are expressed as mean \pm SEM. Differences between 2 groups were assessed using the unpaired two-tailed $t$ test and among more than 2 groups by ANOVA. Data involving more than 2 repeated measures (glucose and insulin tolerance tests, fat loading test, in vivo VLDL-triglyceride production) were assessed by repeated-measures ANOVA. When a significant difference was found with ANOVA, post hoc analyses were performed with Fisher's protected least significant difference test. Differences were considered significant at $P<0.05$. Analyses were performed using Statview Software (BrainPower Inc.).

\section{Acknowledgments}

The authors thank S.J. Fisher and J.K. Kim for helpful advice; H. Masuzaki, E. Hadro, T. Graham, and K. Asakura for expert assistance; M. Trujillo and P.E. Scherer for determining the proportions of highmolecular-weight adiponectin; C.R. Kahn for providing the MCKCre mice; T. Martin for providing comments on the manuscript; and K.C. Hayes and the staff of the Foster Biomedical Research Laboratory at Brandeis University (Waltham, Massachusetts, USA) for excellent care of the animals. We also thank the following investigators for reagents: H.C. Haspel, B. Thorens, L. Rossetti, I. Shimomura, and M.A. Magnuson. This work was supported by NIH grant RO143051 (to B.B. Kahn). K. Kotani is the recipient of a research fellowship from the Japan Science and Technology Corporation.

Received for publication February 16, 2004, and accepted in revised form September 21, 2004.

Address correspondence to: Barbara B. Kahn, Division of Endocrinology, Diabetes and Metabolism, Beth Israel Deaconess Medical Center, 99 Brookline Avenue, Boston, Massachusetts 02215, USA. Phone: (617) 667-5422; Fax: (617) 667-2927; E-mail: bkahn@bidmc.harvard.edu.

Olivier Boss's present address is: 770 Boylston Street, Boston, Massachusetts 02199, USA.

Odile D. Peroni and Yasuhiko Minokoshi contributed equally to this work.
1. Bell, G.I., and Polonsky, K.S. 2001. Diabetes mellitus and genetically programmed defects in betacell function. Nature. 414:788-791.

2. Shepherd, P.R., and Kahn, B.B. 1999. Glucose transporters and insulin action--implications for insulin resistance and diabetes mellitus. N. Engl. J. Med. 341:248-257.

3. Kahn, B.B., and Rossetti, L. 1998. Type 2 diabetes - who is conducting the orchestra? Nat. Genet. 20:223-225.

4. Kitamura, T., Kahn, C.R., and Accili, D. 2003 Insulin receptor knockout mice. Annu. Rev. Physiol. 65:313-332.

5. Minokoshi, Y., Kahn, C.R., and Kahn, B.B. 2003 Tissue-specific ablation of the GLUT4 glucose transporter or the insulin receptor challenges assumptions about insulin action and glucose homeostasis. J. Biol. Chem. 278:33609-33612.

6. Minokoshi, Y., et al. 2002. Leptin stimulates fattyacid oxidation by activating AMP-activated protein kinase. Nature. 415:339-343.

7. Kelley, D.E., and Mandarino, L.J. 2000. Fuel selection in human skeletal muscle in insulin resistance: a reexamination. Diabetes. 49:677-683.

8. Abel, E.D., et al. 2001. Adipose-selective targeting of the GLUT4 gene impairs insulin action in muscle and liver. Nature. 409:729-733.

9. Zisman, A., et al. 2000. Targeted disruption of the glucose transporter 4 selectively in muscle causes insulin resistance and glucose intolerance. Nat.

\section{Med. 6:924-928.}

10. Leloup, C., et al. 1996. Discrete brain areas express the insulin-responsive glucose transporter GLUT4 Brain Res. Mol. Brain Res. 38:45-53.

11. Chin, E., Zhou, J., and Bondy, C. 1993. Anatomical and developmental patterns of facilitative glucose transporter gene expression in the rat kidney. J. Clin. Invest. 91:1810-1815.

12. Katz, E.B., Stenbit, A.E., Hatton, K., DePinho, R., and Charron, M.J. 1995. Cardiac and adipose tissue abnormalities but not diabetes in mice deficient in GLUT4. Nature. 377:151-155.

13. Stenbit, A.E., et al. 1997. GLUT4 heterozygous knockout mice develop muscle insulin resistance and diabetes. Nat. Med. 3:1096-1101.

14. Shulman, G.I. 2000. Cellular mechanisms of insulin resistance. J. Clin. Invest. 106:171-176.

15. Horton, J.D., Goldstein, J.L., and Brown, M.S. 2002. SREBPS: activators of the complete program of cholesterol and fatty acid synthesis in the liver. J. Clin. Invest. 109:1125-1131. doi:10.1172/JCI200215593.

16. Sankar, R., Thamotharan, S., Shin, D., Moley, K.H., and Devaskar, S.U. 2002. Insulin-responsive glucose transporters-GLUT8 and GLUT4 are expressed in the developing mammalian brain. Brain Res. Mol. Brain Res. 107:157-165.

17. Rossetti, L., et al. 1997. Peripheral but not hepatic insulin resistance in mice with one disrupted allele of the glucose transporter type 4 (GLUT4) gene. J. Clin. Invest. 100:1831-1839.
18. Postic, C., et al. 1999. Dual roles for glucokinase in glucose homeostasis as determined by liver and pancreatic beta cell-specific gene knock-outs using Cre recombinase. J. Biol. Chem. 274:305-315.

19. O’Doherty, R.M., Lehman, D.L., Telemaque-Potts, S., and Newgard, C.B. 1999. Metabolic impact of glucokinase overexpression in liver: lowering of blood glucose in fed rats is accompanied by hyperlipidemia. Diabetes. 48:2022-2027.

20. Grimsby, J., et al. 2003. Allosteric activators of glucokinase: potential role in diabetes therapy. Science. 301:370-373.

21. Basu, A., et al. 2000. Effects of type 2 diabetes on the ability of insulin and glucose to regulate splanchnic and muscle glucose metabolism: evidence for a defect in hepatic glucokinase activity. Diabetes. 49:272-283.

22. Basu, A., et al. 2001. Type 2 diabetes impairs splanchnic uptake of glucose but does not alter intestinal glucose absorption during enteral glucose feeding: additional evidence for a defect in hepatic glucokinase activity. Diabetes. 50:1351-1362.

23. Basu, R., Basu, A., Johnson, C.M., Schwenk, W.F., and Rizza, R.A. 2004. Insulin dose-response curves for stimulation of splanchnic glucose uptake and suppression of endogenous glucose production differ in nondiabetic humans and are abnormal in people with type 2 diabetes. Diabetes. 53:2042-2050.

24. Foufelle, F., and Ferre, P. 2002. New perspectives in 
the regulation of hepatic glycolytic and lipogenic genes by insulin and glucose: a role for the transcription factor sterol regulatory element binding protein-1c. Biochem. J. 366:377-391.

25. Aarsland, A., Chinkes, D., and Wolfe, R.R. 1996. Contributions of de novo synthesis of fatty acids to total VLDL-triglyceride secretion during prolonged hyperglycemia/hyperinsulinemia in normal man. J. Clin. Invest. 98:2008-2017.

26. Ruderman, N.B., Richards, K.C., and Valles de Bourges, V. 1968. Regulation of production and release of lipoprotein by the perfused rat liver. J. Lipid Res. 9:613-619.

27. Gibbons, G.F., Bartlett, S.M., Sparks, C.E., and Sparks, J.D. 1992. Extracellular fatty acids are not utilized directly for the synthesis of very-lowdensity lipoprotein in primary cultures of rat hepatocytes. Biochem. J. 287:749-753.

28. Patti, M.E., et al. 2003. Coordinated reduction of genes of oxidative metabolism in humans with insulin resistance and diabetes: potential role of PGC1 and NRF1. Proc. Natl. Acad. Sci. U. S. A.
100:8466-8471.

29. Mootha, V.K., et al. 2003. PGC-1alpha-responsive genes involved in oxidative phosphorylation are coordinately downregulated in human diabetes. Nat. Genet. 34:267-273.

30. Abel, E.D., et al. 1999. Cardiac hypertrophy with preserved contractile function after selective deletion of GLUT4 from the heart. J. Clin. Invest. 104:1703-1714.

31. Bruning, J.C., et al. 1998. A muscle-specific insulin receptor knockout exhibits features of the metabolic syndrome of NIDDM without altering glucose tolerance. Mol. Cell. 2:559-569.

32. Nagy, T.R., and Clair, A.L. 2000. Precision and accuracy of dual-energy $\mathrm{X}$-ray absorptiometry for determining in vivo body composition of mice. Obes. Res. 8:392-398.

33. Kim, J.K., et al. 2001. Glucose toxicity and the development of diabetes in mice with muscle-specific inactivation of GLUT4. J. Clin. Invest. 108:153-160. doi:10.1172/JCI200110294.

34. Rossetti, L., and Giaccari, A. 1990. Relative con- tribution of glycogen synthesis and glycolysis to insulin-mediated glucose uptake. A dose-response euglycemic clamp study in normal and diabetic rats. J. Clin. Invest. 85:1785-1792.

35. Abe, H., et al. 1998. Hypertension, hypertriglyceridemia, and impaired endothelium-dependent vascular relaxation in mice lacking insulin receptor substrate-1. J. Clin. Invest. 101:1784-1788.

36. Li, X., Catalina, F., Grundy, S.M., and Patel, S. 1996. Method to measure apolipoprotein B-48 and B-100 secretion rates in an individual mouse: evidence for a very rapid turnover of VLDL and preferential removal of B-48- relative to B-100-containing lipoproteins. J. Lipid Res. 37:210-220.

37. Pajvani, U.B., et al. 2003. Structure-function studies of the adipocyte-secreted hormone Acrp30/ adiponectin. Implications fpr metabolic regulation and bioactivity. J. Biol. Chem. 278:9073-9085.

38. Minokoshi, Y., Saito, M., and Shimazu, T. 1986. Sympathetic denervation impairs responses of brown adipose tissue to VMH stimulation. Am. J. Physiol. 251:R1005-R1008. 